\title{
The role of some definite references in linguistic integration
}

\author{
E. EUGENE SCHULTZ, JR. \\ Milligan College, Milligan College, Tennessee 37682 \\ and \\ MICHAEL L. KAMIL \\ Purdue University, Lafayette, Indiana 47907
}

\begin{abstract}
Definite reference increases thematic integration for semantically related sentences (DeVilliers, 1974). Therefore, definite reference should increase linguistic integration in the BransfordFranks integrated recognition paradigm. Two recognition and two recall studies demonstrated that, if anything, indefinite reference increases linguistic integration effects, as measured by existing measures of integration. However, definite reference increases topical clustering in recall. Results cast doubt on the validity of existing measures of linguistic integration and indicate that the tally model of performance in integrated recognition tasks (Reitman \& Bower, 1973) is not totally adequate, either. Results are compatible with the associative model of Anderson and Bower (1973). Topical clustering appears to be a better measure of integrated memory than are existing measures of linguistic integration. Retention of information about the propositional form in which semantically related sentences are presented and integrated memory are not necessarily incompatible.
\end{abstract}

Bransford and Franks (1971) have developed a paradigm in which sentences from four different themes are presented during acquisition. Each theme or "complete idea" consists of four propositions. During testing, subjects are presented with acquisition sentences (OLDS), sentences that are thematically consistent with acquisition sentences but contain combinations of propositions that were not previously presented (NEWS), and sentences that are not thematically consistent with acquisition sentences (NONCASES). A minor variant of this paradigm involves presenting only NEWS and NONCASES during testing. Bransford and Franks have found that subjects are unable to discriminate between OLD and NEW sentences, but are able to reject NONCASES during recognition testing. A linear relationship between the number of propositions contained in thematically correct test sentences and summed confidence ratings is also observed. Bransford and Franks argue from these results that memory representations are: (1) semantic, since NONCASES are rejected, and (2) holistic or integrated, because of the linear effect, and because OLDS cannot be discriminated from NEWS.

This research was supported in part by funds from the Purdue University Department of Education. A portion of the experimental results were reported at the 17th Annual Meeting of the Psychonomic Society, St. Louis, Missouri, November 1976. Requests for reprints should be sent to E. Eugene Schultz, Jr., Department of Psychology, Milligan College, Milligan College, Tennessee 37682.
DeVilliers (1974) has demonstrated that the use of definite articles in sentences facilitates learners' perceptions that sentences are connected into a theme. The sentences that DeVilliers used were not unrelated to each other, but rather were related to the extent that they formed a story when definite articles were used. Learners who perceived thematic relatedness among sentences recalled more words and sentences than did learners who did not perceive a theme. DeVilliers argues that definite articles facilitate the relating of nouns to a single referent, making sentences seem more thematic.

Haviland and Clark (1974) have noted that the sentences used by Bransford and Franks (1971) contain only definite articles, which may encourage subjects to assume that there is only one of each object referred to by each noun. Thus, subjects are likely to see that sentences containing common nouns are related to each other, and hence are likely to establish unified representational structures.

The use of indefinite reference in the BransfordFranks integrated recognition paradigm should affect linguistic integration, $\cdot$ as defined by Bransford and Franks (1971). Subjects who are presented with indefinite reference should have more difficulty perceiving that sentences derived from the same theme are related to each other than should subjects who are presented with definite reference. For example, consider the following sentences: (1) Some girl broke some window on some porch. (2) Some window was large. Subjects may have difficulty realizing that Sentences 1 and 2 are thematically related, especially if Sentences 1 
and 2 are not contiguous. (In the Bransford-Franks integrated recognition paradigm, sentences from the same theme are not presented in temporal succession.) However, Sentences 3 and 4 may be readily perceived as thematically related: (3) The girl broke the window on the porch. (4) The window was large. Since indefinite reference inhibits perception of thematicity, representations should be less integrated and holistic if integration effects are demonstrated in the Bransford-Franks integrated recognition paradigm. Thus, subjects who are presented with indefinite reference should demonstrate: (1) better ability to remember the propositional form in which ideas are presented during acquisition, since Bransford and Franks (1971) state that holistic memory and memory for propositional input do not co-occur; and/or (2) a less steep slope for the relationship between the number of propositions contained in thematically correct test sentences and the proportion of positive recognition responses, because differential responding as a function of propositional complexity of sentences indicates the degree to which memory is integrated.

An alternative hypothesis is that linguistic integration effects are produced by strategies subjects employ in the Bransford-Franks integrated recognition paradigm. Subjects may tally the number or proportion of long and short acquisition sentences and use this knowledge to reject a higher proportion of short test sentences when they discover that there are proportionately more short test sentences than long test sentences (Flagg, 1976; Griggs \& Keen, 1977; Katz, Atkeson, \& Lee, 1974; Reitman \& Bower, 1973). According to this view, semantic variables should not affect performance in the Bransford-Franks integration recognition paradigm, so long as the relative proportion of short and long sentences is not changed. Thus, definiteness of reference should not affect subjects' responses on the recognition test.

\section{EXPERIMENT 1}

In this experiment, the above hypotheses were tested in the Bransford-Franks integrated recognition paradigm. According to the integration hypothesis, subjects who are presented with definite reference should integrate information to a greater extent than should subjects who receive indefinite reference. The former should therefore respond with a greater percentage of incorrect responses to NEW recognition sentences and should demonstrate a steeper slope for the relationship between number of propositions in test sentences and positiveness ("oldness") of recognition responses. The tally hypothesis predicts no effects as a function of definiteness of reference. The proportion of positive recognition responses rather than summed confidence ratings should be examined, because James, Hillinger, and Murphy (1977) have shown that summed confidence ratings are somewhat unreliable and insensitive to semantic variables in the Bransford-Franks integrated recognition paradigm.

\section{Method}

Subjects. Seventy-two students enrolled in introductory psychology at Purdue University participated in this study. Course credit was given for participation.

Stimuli and Design. Sentences in the definite reference condition were exactly the same as those in Experiment 2 of Bransford and Franks (1971). In the indefinite reference condition, every "the" was changed to "some." The reason that "some" was used in this particular study is that its use eliminated the necessity of using "a" as an article in some sentences and "an" in other sentences. Thus, minimal changes to sentences originally used by Bransford and Franks had to be made.

Acquisition sentences contained exactly eight ONES (sentences that contained one idea), eight TWOS, and eight THREES. Test sentences contained eight ONES, eight TWOS, four THREES, four FOURS, and six NONCASES. All test sentences were new, as in Experiment 2 of Bransford and Franks (1971). NONCASES expressed incorrect relationships between ideas contained in acquisition sentences. The presentation order of both acquisition and test sentences, and of elliptical questions that were asked after the presentation of each acquisition sentence, was randomly determined. Elliptical questions were similar to those used in Experiment 2 in Bransford and Franks (1971). For example, the question for Sentence 3 was "Broke what?"

Half of the subjects were randomly assigned to the indefinite reference condition; the others were assigned to the definite reference condition. The other independent variable was propositions in test sentences (ONES, TWOS, THREES, and FOURS). The experimental design involved one between-subjects variable (definiteness of reference) and one within-subjects variable (sentence complexity).

Procedure. Two experimental sessions, one for each betweensubjects treatment condition, were held in a classroom. Subjects were told that a series of sentences would be presented, one sentence at a time, and that a short question about the sentence would be asked immediately after each sentence was presented. Subjects were instructed to answer each question silently, then to listen to the next sentence, and so on, until all sentences had been read. The experimenter read a sentence slowly, paused for approximately $2 \mathrm{sec}$, asked a question, then paused for approximately $3 \mathrm{sec}$, read the next sentence, and so on.

After the last acquisition sentence and question had been read, each subject was given a test sheet upside down. After a retention interval of $20 \mathrm{sec}$, subjects were instructed to turn over the test sheet, read the instructions, and begin working.

Instructions with the test sentences read: "Now read each sentence below and decide if it is a sentence which was presented previously. If it is, mark OLD, and if not, mark NEW. Cover each sentence after you have made your response to it." Subjects were self-paced during testing. A maximum of $8 \mathrm{~min}$ was allowed for testing. All subjects finished the task within this limit.

\section{Results}

The dependent variable was the proportion of OLD responses. Analysis of variance for the effect of definiteness of reference and sentence complexity revealed a statistically significant main effect of reference $[F(1,70)=6.03, p<.025]$. Indefinite reference produced a greater proportion of OLD recognition responses (mean $=.61$ ) than did definite reference (mean $=.53$ ). The only other statistically significant effect was the main effect of sentence complexity 
$[F(3,210)=25.37, p<.001]$. A linear trend test (Winer, 1971) demonstrated that the relationship between number of propositions in test sentences and proportion of OLD responses was linear $[F(1,210)=14.27$, $\mathrm{p}<.001$ ] (see Figure 1). The Reference by Sentence Complexity interaction was not significant $[F(3,210)<1$, $p>.05]$. A linear slope test (Winer, 1971) revealed that the slope of proportion of OLD responses as a function of sentence complexity did not differ reliably for the definite and indefinite reference conditions $[\mathrm{F}(1,210)<1, \mathrm{p}>.05]$.

A second analysis of variance showed that there was no significant difference in proportion of OLD responses for NONCASES as a function of reference $[F(1,70)<1$, $\mathrm{p}>.05]$. False alarm rates for the definite and indefinite reference conditions were .03 and .06 , respectively.

\section{Discussion}

The Bransford-Franks linear effect was replicated. However, the results are not entirely consistent with predictions derived from either the tally or the integration hypotheses. Subjects who received indefinite reference had a higher false alarm rate for NEW test sentences, contrary to what was predicted by either hypothesis. Slopes for the relationship between sentence complexity and proportion of positive recognition responses did not differ as a function of reference. These results suggest that, contrary to what might be expected on the basis of findings by DeVilliers (1974), definite reference does not facilitate integrated recognition memory, as defined by Bransford and Franks (1971). In fact, the finding that indefinite reference produced a higher false alarm rate could even be interpreted to indicate that indefinite reference somehow increased linguistic integration, since integrated storage and retention of the exact propositional form of



Figure 1. Mean proportion of OLD recognition responses for sentences with definite references (filled circles) and indefinite references (open circles) in Experiment 1. sentences are postulated by Bransford and Franks (1971) to be at the opposite ends of a continuum.

Thus, it is difficult to conceive how the results of Experiment 1 can be attributed to processes of integrated memory; definite reference should have produced a greater amount of linguistic integration, according to the integration hypothesis. If subjects simply tally the number of short and long acquisition and test sentences, then the particular reference used should make no difference during recognition testing, according to the tally model. However, references did affect recognition responding. A further study was conducted to clarify these results by examining other possible explanations of these results.

\section{EXPERIMENT 2}

In Experiment 1, there were some procedural variations that may have affected the results. Subjects were not instructed to write down answers presented during acquisition. Although Curnow's (1969) research makes this possibility unlikely, perhaps having subjects answer questions silently "tipped them off" to the fact that the questions were unimportant, and not the focus of the study. Confidence ratings were not taken. Although James et al. (1977) have shown confidence ratings to be an inferior measure to the proportion of positive responses in the Bransford-Franks integrated recognition paradigm, the question remains whether results can be replicated using confidence ratings. In addition, no OLD sentences were presented in Experiment 1. The possible objection to the use of the indefinite reference "some" in this recognition paradigm could also be answered if "a" were used in a replication of Experiment 1.

\section{Method}

Subjects. Sixty students enrolled in an introductory psychology course at Milligan College served as subjects. Participation in this experiment partially fulfilled an experimental participation requirement in this course.

Procedure. Half of the subjects were randomly assigned to the definite reference condition, and half were randomly assigned to the indefinite reference condition. Subjects were told that a series of sentences would be presented, one sentence at a time, and that a short question about each sentence would be asked immediately after the sentence was presented. Each subject was then handed a piece of paper in which 30 numbers appeared in a vertical column. Subjects were instructed to write down the answer to each question on this piece of paper as each question was asked.

The experimenter then read an acquisition sentence slowly, paused for approximately $2 \mathrm{sec}$, asked a question, then paused for approximately $5 \mathrm{sec}$ ( to allow time for writing down the one or two words needed to answer each question), read the next sentence, and so on. After presentation of the last acquisition sentence and question, a test sheet was presented upside down. After a retention interval of $20 \mathrm{sec}$, subjects were told to turn over the test sheet, read the instructions, and start the recognition task. Instructions read as follows: "Now read each sentence below and decide if it is a sentence which was presented previously. If it is, mark OLD, and if not, mark NEW. Then immediately indicate your degree of confidence in your choice 
by circling a number between 1 and 5 next to the OLD-NEW alternative for that sentence. Circle 1 if you are very unsure of your response, 5 if you are very sure, and a number in between to express intermediate degrees of confidence. Cover each sentence after you have made your responses to it." Subjects were self-paced during testing, as in Experiment 1. Again, $8 \mathrm{~min}$ were allowed for testing, and all subjects were through before this time period expired.

\section{Results}

For NONCASES, mean weighted confidence ratings were computed for each subject. Analysis of variance for NONCASES revealed a statistically significant effect of reference $[F(1,58)=4.98, p<.05]$ when the dependent variable was mean summed confidence ratings. Out of a possible range of mean weighted confidence ratings from -5 to +5 , subjects who were presented with definite reference obtained a mean weighted confidence rating of -4.64 . Subjects who were presented with indefinite reference produced a mean weighted confidence rating of -4.25 . Similar results were obtained when the dependent variable was number of correct responses to NONCASES $[\mathrm{F}(1,58)=4.50$, $\mathrm{p}<.05]$. The mean number of correct responses for the definite and indefinite reference conditions was 5.93 and 5.73 , respectively, out of a possible maximum score of 6 .

Signal detection measures were used to analyze performance on OLD and NEW sentences. Area under the operating characteristic was calculated, then converted to $\mathrm{d}^{\prime}$ scores for the definite and indefinite reference conditions. Analysis of variance revealed that memory discriminability was greater for the definite reference $\left(d^{\prime}=.11\right)$ than for the indefinite reference $\left(d^{\prime}=.04\right)$ condition $[F(1,58)=8.18, p<.01]$. The mean proportion of OLD responses in both conditions was .64 . This proportion was above the .50 proportion expected by chance in the definite reference condition $\left[\chi^{2}(1)=95.91, p<.001\right]$ and in the indefinite reference condition $\left[\chi^{2}(1)=89.42, p<.001\right]$, indicating that subjects had a positive response bias when responding (cf. Soli \& Balch, 1976).

Another analysis of variance was then performed for mean weighted confidence ratings as a function of reference, type of sentence (OLD or NEW), and number of propositions (one, two, or three). The main effect of number of propositions was highly significant $[F(1,116)=120.54, p<.001]$. Mean confidence ratings for ONES, TWOS, and THREES were -.61, 1.69, and 2.90 , respectively. A linear trend test (Winer, 1971) demonstrated that the relationship between number of propositions in test sentences and mean weighted confidence ratings was linear $[\mathrm{F}(1,116)=58.29$, $p<.001]$. The main effect of type of sentence was also highly significant $[F(1,58)=26.05, p<.001]$. The mean weighted confidence rating for OLD sentences was 1.64 , whereas the mean weighted confidence rating for NEW sentences was 1.02. Two interactions were also statistically reliable: (1) the Type of Sentence by Type of Reference interaction $[F(1,58)=5.20, p<.05]$, and (2) the Sentence Complexity by Type of Sentence interaction $[F(2,116)=18.30, p<.001]$ (see Figure 2). A linear slope test (Winer, 1971) demonstrated that the slope for the mean weighted confidence ratings as a function of sentence complexity did not significantly differ for the definite and indefinite reference conditions $[F(1,116)<1, p>.05]$.

\section{Discussion}

Again, the Bransford-Franks linear effect was replicated. The results of Experiment 2 differ somewhat from the results of Experiment 1, in that in Experiment 2, subjects who were presented with definite reference did better in rejecting NONCASES than did subjects who were presented with indefinite reference. However, these results are not central to the issue addressed in this paper, since Bransford and Franks (1971) argue that the rejection of NONCASES indicates that subjects abstract the "gist" of ideas presented in acquisition sentences. Thus these results suggest that representations for sentences with definite reference were more semantically precise (cf. Bransford \& Franks, 1972 ), which is consistent with the results of DeVilliers' (1974) study.

However, other results are consistent with the results in Experiment 1. Reference did not affect the slopes of the relationship between weighted confidence ratings and sentence complexity, indicating that reference did not affect the degree to which representations were integrated, assuming that slope is a valid measure of memory integration. When signal detection analysis was employed to separate response biases from memorial processes, it was found that subjects who were presented with definite reference were better able to discriminate between OLDS and NEWS. Subjects presented with

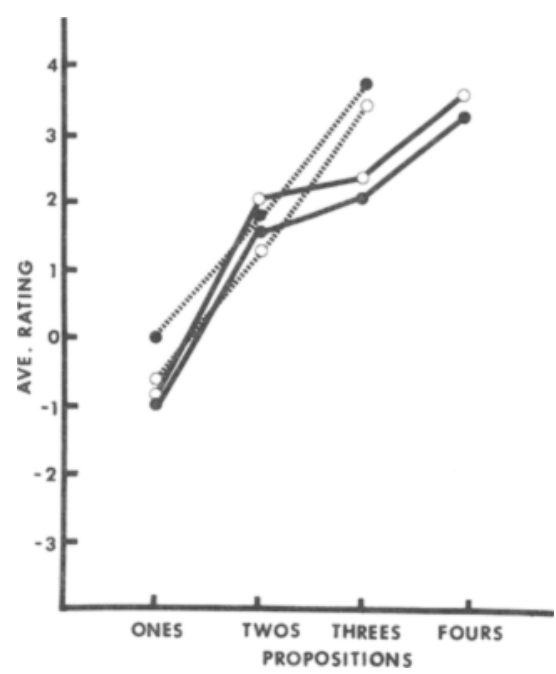

Figure 2. Mean weighted confidence ratings for OLD (dotted lines) and NEW (solid line) sentences with definite (filled circles) and indefinite (open circles) references in Experiment 2. 
indefinite reference were less able to discriminate between OLDS and NEWS, however. The main effect of type of sentence (OLDS or NEWS) and the interaction between type of sentence and sentence complexity replicate findings for a condition in which test sentences were presented visually in a study by Flagg and Reynolds (1977).

Again, these results do not support the integration hypothesis. Reference should have affected in tegrated recognition, as evidenced by a change in slope between confidence ratings and sentence complexity. The finding that definite reference produces greater ability to discriminate OLDS from NEWS than does indefinite reference is contrary to the notion that integrated recognition memory is characterized by inability to recognize specific input sentences (cf. Bransford \& Franks, 1971). If anything, one might then argue that indefinite reference leads to more holistic representation.

However, these data also appear to present difficulties for the tally model (Reitman \& Bower, 1973). As previously stated, the proportion of short and long acquisition and test sentences is unchanged by manipulating definiteness of reference. It is not immediately apparent how a tally model can account for the effect of reference upon discriminability of OLDS from NEWS and the Type of Reference by Type of Sentence interaction in Experiment 2.

\section{EXPERIMENT 3}

DeVilliers' (1974) study measured recall, not recognition. Perhaps the thematically unifying effects of definite reference occur only when a great deal of retrieval is necessary, as in recall, but not in recognition (Bernbach \& Kupchak, 1972). Experiment 3 was similar to Experiments 1 and 2, except that recall was measured in Experiment 3. If definite reference increases linguistic integration, then subjects who are presented with definite reference should recall proportionately more nonpresented propositional combinations than should subjects who are presented with indefinite reference. No test of the tally model can be made in recall, however, since the tally model deals with performance strategies in recognition testing.

\section{Method}

Subjects. Forty students enrolled in an introductory psychology course at Purdue University volunteered to participate. No subject had participated in any previous experiment.

Stimuli and Procedure. The procedures for the acquisition phase of this experiment, including instructions, were identical to those used in Experiment 1. Sentences were those used as acquisition sentences in Experiment 1. Sentences with either definite or indefinite reference were presented auditorily in the same order as in Experiment 1. Again, a question was asked after each sentence was read. Questions were also those used in Experiment 1. After a 20 -sec retention interval, subjects were instructed to recall sentences the way the sentences were presented as precisely as possible. However, they were told that it was not necessary to attempt to recall sentences in the order of presentation. Subjects were allowed $7.5 \mathrm{~min}$ for recall. Subjects were randomly assigned to treatment conditions.

\section{Results}

Protocols were scored for recall of: (1) OLDSsentences containing exact combinations of ideas that were presented in acquisition sentences; (2) NEWSsentences containing idea combinations not presented in acquisition sentences, but expressing ideas for each theme correctly; and (3) NONCASES-sentences inconsistent with themes presented during acquisition. For example, both of the following sentences was judged as OLD, because each contains the same idea combinations as in Sentence 3: (5) The girl had broken the porch window. (6) The window on the porch was broken by the girl. The following was judged as NEW, because all ideas had not been previously presented together during acquisition: (7) The girl who lives next door broke the window on the porch. The following was considered a NONCASE: (8) The dog broke the window on the porch.

Analysis of variance was performed for the proportion of NEWS to NEWS + OLDS generated, a measure of memory integration. Type of reference was an independent variable in this and all subsequent analyses. Inspection of data revealed that subjects varied greatly in the proportion of NEWS generated, indicating that some subjects were more "liberal" in generating NEWS than others. A second independent variable, "liberal" vs. more "conservative" generation, was thus also introduced by separating the 10 most "liberal" from the other 10 more "conservative" subjects in each level of the definiteness-of-reference variable. A highly significant effect of type of reference was found $[F(1,36)=12.04$, $\mathrm{p}<.005]$. Subjects who received definite reference generated a smaller mean proportion (mean $=.34$ ) of NEW sentences than did subjects who received indefinite reference $($ mean $=.46)$. The generation variable was also highly significant $[F(1,36)=54.71, p<.001]$, indicating that "liberals" (mean $=.53)$ generated proportionately more NEWS than did "conservatives" $($ mean $=.27)$. The interaction of the two independent variables was not statistically significant $[F(1,36)<1$, $\mathrm{p}>.05]$

A second analysis of variance revealed that there was no statistically significant difference in the number of NONCASES recalled as a function of type of reference $[\mathrm{F}(1,38)<1, \quad \mathrm{p}>.05]$. The mean number of NONCASES generated in the definite and indefinite reference conditions was .77 and .86 , respectively. A third and fourth analysis of variance demonstrated that definite reference produced greater generation of OLDS $[F(1,38)=10.30, p<.01]$, but not NEWS $[F(1,38)=1.23, p>.05]$. Mean recall of OLDS for the definite and indefinite reference conditions was 6.15 and 3.71, respectively. Mean recall of NEWS for these conditions was 3.31 and 2.57 , respectively. 


\section{DISCUSSION}

These results parallel the results obtained for the recognition of semantically related sentences in Experiments 1 and 2. Subjects who were presented with definite reference actually generated proportionately fewer nonpresented propositional combinations than did subjects presented with indefinite reference. This finding is again contrary to what is predicted by the integration hypothesis. Clearly, there is no support for the hypothesis that definite reference increases memory integration in either recognition or recall of semantically related sentences. Indeed, definite reference appears to decrease memory integration. However, the work of DeVilliers (1974) would suggest exactly the opposite, since definite articles increase subjects' ability to perceive thematic interrelatedness of sentences.

\section{EXPERIMENT 4}

One might argue that the results obtained in Experiment 3 were due to the use of the reference "some." Again, a critic might argue that this reference made sentences anomalous, and thus more difficult to comprehend. Thus, the effects in Experiment 3 may have been due to variables related to comprehension, and not integration. Another experiment was run to examine this possibility. In this experiment, the iridefinite reference was changed to determine whether the particular reference used in Experiment 3 was responsible for the results obtained.

\section{Method \\ Subjects. Forty-eight students enrolled in an introductory educational psychology course at Purdue University participated in this study. Course credit was given for participation. No subject had participated in any of the previous experiments. \\ Stimuli and Procedure. All aspects of this experiment were the same as in Experiment 3, with the following exceptions: (1) In the sentences with indefinite reference, each "some" was changed to "a" and each "old" was changed to "small." (2) Twenty-four subjects were presented with definite reference, and 24 were presented with indefinite reference.}

\section{Results and Discussion}

Scoring and analyses of variance were performed in a manner identical to that reported in Experiment 3. Again, definite reference produced a highly significant decrement in proportion of NEWS recalled $[F(1,44)=9.12, p<.005]$. The mean proportion of NEWS recalled for the definite and indefinite reference conditions was .30 and .40 , respectively. Again, "liberal" subjects generated proportionately more NEWS (mean $=.48$ ) than did more "conservative" subjects (mean $=.22)[F(1,44)=68.30, p<.001]$. The interaction between independent variables was not statistically significant $[F(1,44)=1.45, p>.05]$. The mean number of NONCASES generated in the definite and indefinite reference conditions was 2.00 and 2.46 , respectively.
Again, subsequent analyses of variance revealed that definite reference produced greater generation of OLDS $[F(1,46)=4.55, p<.05]$, but not NEWS $[F(1,46)=2.15, p<.05]$. Mean recall of OLDS for the definite and indefinite reference conditions were 6.35 and 4.55 , respectively. Mean recall of NEWS for these conditions was 3.31 and 2.57 , respectively.

These results replicate the results obtained in Experiment 3. Thus, the effects reported in Experiment 3 are not due to the use of the particular reference "some."

\section{GENERAL DISCUSSION}

All four studies in this paper have failed to substantiate that definite reference produces a greater amount of integrated memory when measures of linguistic integration are used. Indefinite reference might actually produce more holistic representation, as indicated by one measure of linguistic integration. Because these findings are counterintuitive, one might question whether the use of definite reference fosters thematic integration in conditions such as those in the studies in this paper. However, several findings from Experiments 3 and 4 suggest that the effects of definite reference in semantically related sentences are within the boundary conditions of the effects obtained by DeVilliers (1974). First, in Experiments 3 and 4, definite reference increased recall of acquisition sentences. These results are strikingly parallel to DeVilliers' results. Second, a post hoc analysis of data from Experiments 3 and 4 revealed that topical clustering increased when definite reference was presented (see Table 1). Topical clustering is a measure of the number of topically related sentences that are recalled contiguously (cf. Rickards, 1976). For example, if sentences from different themes are presented during acquisition, each thematically correct pair of sentences from one theme that is recalled successively is considered one topical cluster. A thematically incorrect sentence between two thematically correct sentences from one theme precludes an increase in the clustering score for the thematically correct sentences, as does a sentence from a different theme between two thematically correct sentences from one theme.

In Experiments 3 and 4, topical clusters were defined as two contiguously recalled OLDS from the same theme. Thus, the recall of a NEW sentence from any

Table 1

Topical Custering Scores for Sentences With Definite and Indefinite References (Experiments 3 and 4)

\begin{tabular}{cccccc} 
& \multicolumn{2}{c}{$\begin{array}{c}\text { Experiment } 3 \\
\text { ("the"vs. "some") }\end{array}$} & & \multicolumn{2}{c}{$\begin{array}{c}\text { Experiment } 4 \\
\text { ("the" vs. "a") }\end{array}$} \\
\cline { 2 - 3 } \cline { 5 - 6 } Type of & OLDS & OLDS + & & OLDS & OLDS + \\
Reference & Only & NEWS & & Only & NEWS \\
\hline Definite & 3.85 & 4.69 & & 3.21 & 4.34 \\
Indefinite & 1.14 & 2.01 & & 1.71 & 2.68 \\
\hline
\end{tabular}


theme precluded the possibility of a cluster between the two sentences contiguous to the NEW sentence. In Experiment 3, the difference in topical clustering as a function of reference was statistically significant $[F(1,38)=8.01, p<.01]$ (see Table 1). Similar results were obtained in Experiment $4 \quad[F(1,46)=4.54$, $p<.05]$. Results were similar when topical clusters were redefined as any combination of OLDS and NEWS recalled contiguously from one theme. Again, topical clustering was affected by reference in Experiment 3 $[F(1,38)=7.50, \quad p<.025]$ and in Experiment 4 $[F(1,46)=4.17, p<.05]$ (see Table 1). The increase in topical clustering produced by definite reference demonstrates that subjects who received definite references recalled sentences in a more thematically interrelated manner. Again, these results show that the manipulations in these experiments are within the boundary conditions of the DeVilliers (1974) effects, and that, in reality, definite reference produced greater thematic integration.

The juxtaposition of more thematic integration and less linguistic integration thus becomes of paramount importance. Does definite reference actually lead to less linguistic integration but more thematic integration? This possibility seems unlikely, in that both types of integration should be highly interrelated. Because only data based upon a measure of thematic integration were consistent with previous findings (DeVilliers, 1974), measures of linguistic integration must be brought into question. A likely possibility is that presenting semantically related sentences, then testing for retention of exact propositional combinations of ideas expressed in these sentences (Bransford \& Franks, 1971) does not yield valid measures of memorial integration. If measures of linguistic integration derived from such a procedure are valid, why does indefinite reference apparently increase linguistic integration, as shown in all four studies reported in this paper? Numerous other studies (Flagg, 1976; Griggs \& Keen, 1977; Katz et al., 1974; Reitman \& Bower, 1973) also cast doubt upon the adequacy of the Bransford-Franks recognition paradigm to indicate the degree to which representations are integrated.

The tally model does not seem totally adequate to account for results obtained in Experiments 1 and 2, in which recognition was measured. The tally model predicts that semantic variables such as definiteness of reference should not affect recognition performance, so long as the relative proportion of short and long acquisition and test sentences is unchanged. The findings that definiteness of reference affects the relationship between measures of linguistic integration and sentence complexity indicates that semantic variables such as definiteness of reference must be accounted for in strategy explanations of performance in the Bransford-Franks integrated recognition paradigm.

An adequate account of processes occurring in memory for semantically related sentences must be able to explain: (1) the slope for the relationship between sentence complexity and recognition responses, (2) the ability of subjects to reject NONCASES, (3) the ability of subjects to discriminate to some extent between OLD and NEW sentences in recognition and to recall a greater proportion of acquisition sentences in recall, and (4) the increase in topical clustering as the result of presentation of definite reference. A model that appears capable of dealing with all of these results is the associative model (HAM) proposed by Anderson and Bower (1973). The HAM model has already dealt with the first two results (Anderson \& Bower, 1973, pp. 347. 352). Findings 3 and 4 can be explained by HAM's recognition mechanism. The MATCH process of HAM attempts to establish correspondence between memory structure and input information by matching links from input information to the associative memory structure that is most similar in terms of number of links and sequence of relations labeling the links. The more nodes the MATCH process must search, the less likely the MATCH process is to make a correspondence between input from a test sentence and a segment of associative structure in memory. Because definite reference should direct the MATCH process to one particular concept node (Anderson \& Bower, 1973, p. 392), definite reference should reduce the probability that information is forgotten. Since the MATCH process is more likely to go to one particular node when definite reference is used, and since the MATCH process subsequently will search memory structures accessible from this node, more topically related sentences should be recalled contiguously. NEWS should be recalled, regardless of reference used, because information about contextual links between propositions is difficult to compute and retain. However, proportionately more OLDS should be recalled when definite reference is presented, because context links should intersect more at one particular node than when indefinite reference is presented. Thus, subjects presented with definite reference should be able to discriminate between OLDS and NEWS to a certain extent. To summarize in more general terms, HAM predicts that definite reference results in better memory for individual sentences, and this will make discrimination easier in the recognition memory test.

Because results presented in this paper cast doubt on the adequacy of existing measures of linguistic integration, the problem of deriving a suitable measure of integrated memory needs resolution. It is entirely possible that subjects may integrate information only when integration facilitates retrieval. Because recall requires retrieval, but recognition requires little or none (Bernbach \& Kupchak, 1972), it is theoretically conceivable that a measure of recall such as topical clustering may be more sensitive to integration effects in memory than measures of recognition. Indeed, the 
post hoc analysis of Experiments 3 and 4 demonstrates empirically that topical clustering may be a suitable measure of integration. Topical clustering has been shown to be affected in a manner that is predictable on the basis of current knowledge of thematic integration (cf. DeVilliers, 1974). Schultz and Kamil (in press) have also reported that topical clustering in recall for semantically related sentences increases as a function of (1) thematic information presented before acquisition sentences are presented, and (2) the topical organization of acquisition sentences. Thus, there is some evidence to support the validity of the use of topical clustering as a measure of memory in tegration.

Finally, there is a growing body of evidence that suggests that retention of information about the propositional form in which semantically related sentences are presented and integrated memorial processes are not necessarily incompatible (Lawson, 1977; Schultz \& Kamil, in press). Results presented in this paper are totally supportive of this plausible notion.

\section{REFERENCES}

ANDERson, J. R., \& Bower, G. H. Human associative memory. Washington, D.C: Winston, 1973.

Bernbach, H. A., \& KupchaK, P. G. Recognition and recall in short-term memory. Journal of Mathematical Psychology, 1972, 9. 237-242.

Bransford, J. D., \& Franks, J. J. The abstraction of linguistic ideas. Cognitive Psychology, 1971, 2, 331-350.

Bransford, J. D., \& Franks, J. J. The abstraction of linguistic ideas: A review. Cognition: International Journal of Cognitive Psychology, 1972, 1, 221-249.
CURnow, P. G. Integration of linguistic materials. Unpublished $\mathrm{PhD}$ dissertation, University of Minnesota, 1969.

DEVILLIERS, P. A. Imagery and theme in recall of connected discourse. Journal of Experimental Psychology, 1974, 103, 263-268.

FLAGG, P. W. Semantic integration in sentence memory? Journal of Verbal Learning and Verbal Behavior, 1976, 15, 491-504.

FlagG, P. W., \& Reynolds, A. G. Modality of presentation and blocking in sentence recognition memory. Memory \& Cognition, 1977. 5, 111-115.

GRIGGS, R. A., \& KEEN, D. M. The role of test procedure in linguistic integration studies. Memory \& Cognition, 1977, 5, 685-689.

Haviland, S. E., \& Clark, H. H. What's new? Acquiring new information as a process in comprehension. Joumal of Verbal Learning and Verbal Behavior, 1974, 13, 512-521.

J AMES, C. T., Hillinger, M. L., \& Murphy, B. J. The effects of complexity on confidence ratings in linguistic integration. Memory \& Cognition, 1977, 5, 355-361.

LAWSON, R. Representation of individual sentences and holistic ideas. Journal of Experimental Psychology: Human Learning and Memory, 1977, 3, 1-9.

Katz, S., Atkeson, B., \& LeE, J. The Bransford-Franks linear effect: Integration or artifact? Memory \& Cognition, 1974, 2, 709-713.

Rettman, J. S., \& Bower, G. H. Storage and later recognition of exemplars of concepts. Cognitive Psychology, 1973, 4, 194-206.

Rickards, J. P. Interaction of position and conceptual level of adjunct questions on immediate and delayed retention of text. Journal of Educational Psychology, 1976, 68, 210-217.

Schultz, E. E., \& Kamil, M. L. How integrated is memory for semantically related sentences? Journal of General Psychology, in press.

Soli, S. E., \& BALCH, W. R. Performance biases and recognition memory for semantic and formal changes in connected discourse. Memory \& Cognition, 1976, 4, 673-676.

WINER, B. J. Statistical principles in experimental design (2nd ed.). New York: McGraw-Hill, 1971.

(Accepted for publication December 5, 1978.) 